\title{
Emergency Notification Using Combination Algorithm with Recognition ECG Signal
}

\author{
https://doi.org/10.3991/ijoe.v16i05.12707 \\ Kittimasak Naijit \\ Chandrakasem Rajabhat University, Bangkok, Thailand \\ kittimasak.nechandra.ac.th
}

\begin{abstract}
Intensive Care Unit (ICU) Rooms usually have several detectors attached to each patient providing intensive care, and several processors control and interpret. If the processor detects an abnormality, the medical professional office will be alerted. Nevertheless, many patients with heart disease are concerned with day-to-day behaviors such as hard work, battle, exercise, shock, fight, and war. Become due to clinical depression and erectile impotence this induces anxiety and fear. The boundaries of your heart muscle and coronary strength are unclear. They want a warning that is quick and accurate before they lose control. We develop signal recognition for an algorithm that is very fast and accurate. It helps alert patients to avoid the operation of risk. Nevertheless, it is able to transfer information from the heartbeat network to the doctor's guidance system. The research would analyze the 200 signals from multiple ECG signals. Integration Component Diagnosis (ICD) is capable of extraordinary reliability of identification than the 17.10-41.93 average percentage of the Automata Matching Process which takes less time than the other 29.37 average percentage process and can alert within 12 seconds. It cannot be identified in a pattern other than without the detection of an ECG signal. In the experimental, the signal is used to distinguish 20 ECG signal patterns.
\end{abstract}

Keywords-Emergency notification, combination algorithm, ECG signal

\section{Introduction}

The signal analysis has contributed significantly to the understanding of the Electrocardiogram (ECG) and its dynamic features, as shown by changes in timing and beating structure. [12] For example, techniques have been developed that characterize cardiovascular oscillations and represent subtle cardiovascular variations. [2] The detection of alternating low-level changes in T-wave amplitude was a further example of oscillating behaviour, [17] which have been identified to show increased risk of sudden lifethreatening arrhythmias. [18] None of these two oscillatory signal properties can be detected by the naked eye from a standard ECG print. A simple collection of algorithms that monitor the signal for different types of noise and disturbances [8][22], control heartburn, and gather basic ECG measurements of wave amplitudes and length and 
compact data for efficient processing are standard for all ECG methods of study, regardless of the ECG diagnosis, stress testing, external surveillance, or intensive care surveillance. [7] Highly inadequate pumping capacity for blood flow controls is used to meet the requirements of the body, also known as congestive heart failure. The body, known as congestive heart insufficiency, is using extremely inadequate pumping capacity for blood flow controls.

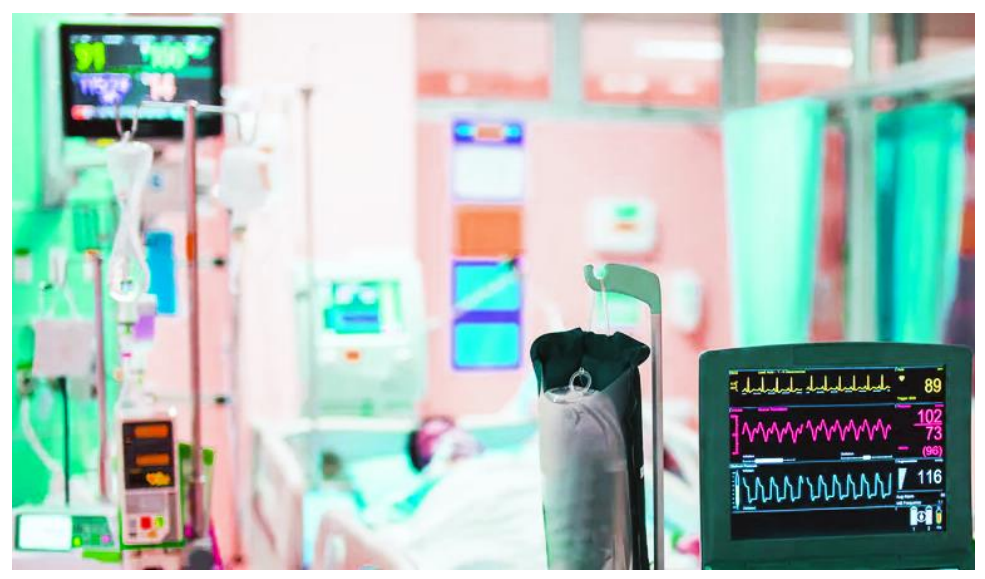

Fig. 1. Patients Risk for Heart Failure

In figure 1, heart failure may result in a number of symptoms including shortness of breath, swelling of the legs, and intolerance to exercise. Echocardiography and blood tests have been associated with the disease. This is a test performed in an office physician where the body surface of the resting patient records 12 different potential differences or ECGs. [15][23] Adding that we use another set of potential body surface as inputs to a three-dimensional vector model of cardiac excitation. The result is a schematic illustration of the excitement of the heart or a cardiogram of the wave. Nevertheless, ECG leads are tracked or recorded for life-threatening variations in the rhythm of the heartbeat for long-term observation in the intensive care unit or on out patients. [4][10] ECG interpretation techniques have been developed initially and are now used in electronic machine computing. The ECG was sent to the device from remote hospital locations using a specially designed ECG acquisition cart that could be transported to the bedside of the patient. [9] As technologies progressed, microcomputers installed within the hospital assumed the role of the remote big computer. [23] The ECG acquisition cart continued to include an integrated microprocessor to enable the processing of the ECG. [21] When the ECG was a noise signal, the interpretation algorithm had an increased failure rate. Through running digital signal processing algorithms, the microprocessor improved the signal to noise ratio to eliminate background drift and attenuate liner interference. This research proposes: 1). A framework for data operation in rapid ECG analysis. 2). A classification algorithm using optimization analysis which is used for the extraction of features and for the recognition of the ECG signal.

This research supports the expert medical system. 


\section{Methodology}

\subsection{Signal combination algorithm}

ECG wave pattern: We use classification methods for the pattern of identification in the ECG signal, which is quite similar to the process of machine recognition. [5] We use cross-correlation patterns to distinguish different signal patterns. The signals associated are the wave pattern forms of two signals that complement each other. The correlation coefficient is a function that specifies the degree of functionality between the two signal shapes of the ECG wave pattern recognition system used by Integration Component Diagnosis (ICD) for pattern recognition.

Automata template matching: We use a series of tokens that would represent a standard ECG. A set of tokens is an interface to the finite state automata. The series of tokens must be derived from the information of the ECG signal. This is achieved by generating a sequence of differences in the input information. The algorithm can now allocate a wave pattern token to each of the groups formed previously based on the values of the number and the sum of the differences in each group. We use ranges of QRS up-wards or down-wards, and then a norm-up or norm-down token is created for that class of differences. The QRS is the combination of three of a typical ECG's graphical deflections. [19] If the values of number and sum do not fall within this range, a noise-up or noise-down token will be created. The zero token is created if the sum for a group of differences is zero. The algorithm reduces the information of the ECG signal to a series of tokens that can be fed to finite state automata for QRS detection. [17] It's a moderate pattern of ECG detection. (Figure 2)

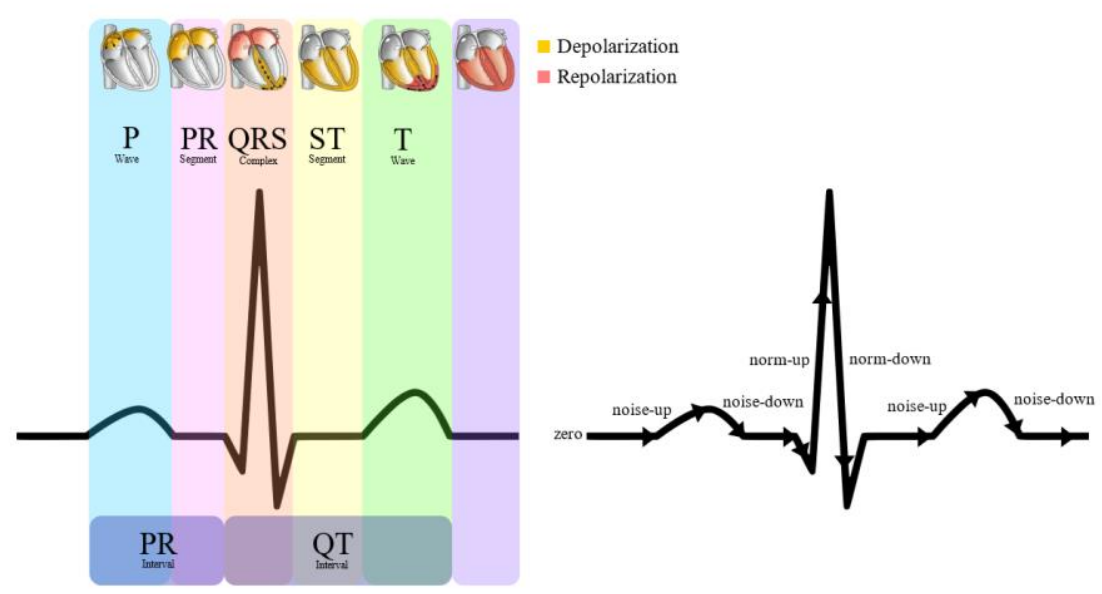

Fig. 2. Automata Template Matching

It is possible to use the QRS polarity in leads I, leads II, and leads III to measure the electrical direction of the heart in the frontal plane. (Table 1) 
Table 1. QRS polarity in leads I, II, and III

\begin{tabular}{|c|c|c|c|c|c|c|}
\hline & $\begin{array}{c}\text { Normal Axis } \\
0 \text { to } 90\end{array}$ & $\begin{array}{c}\text { Left Axis } \\
\text { Physiological } \\
0 \text { to }-30\end{array}$ & $\begin{array}{l}\text { Left Axis } \\
\text { Physiological } \\
-30 \text { to }-90\end{array}$ & $\begin{array}{l}\text { Right Axis } \\
90 \text { to } 180\end{array}$ & $\begin{array}{l}\text { Extreme Axis } \\
-90 \text { to }-180\end{array}$ & $\begin{array}{c}\text { Indeterminate } \\
\text { Axis }\end{array}$ \\
\hline Lead & & & & & & \\
\hline $\begin{array}{c}\text { Lead } \\
\text { II }\end{array}$ & $J \mathrm{~L}$ & & & & & \\
\hline $\begin{array}{c}\text { Lead } \\
\text { III }\end{array}$ & $L$ & & & $\Lambda$ & $V$ & \\
\hline
\end{tabular}

\subsection{Wave pattern of ECG signal recognition}

The ECG is one of all medicine's most common, enduring, and important tests. It is easy to perform, non-invasive, yields tests quickly, and is useful to treat thousands of heart conditions. Lately, the ECG has become even more important because a specific ECG trend, called ST elevation, is a strong indication that there has been a severe heart attack, and more emphasis than ever is placed on handling heart attacks as soon as possible. The ECG will not necessarily be part of a physical routine, but you will almost certainly get an ECG if you need medical attention because you have chest pain, sudden unexplained shortness of breath, or other symptoms that suggest a possible heart attack. (Table 2)

Table 2. Wave Pattern of ECG Signals

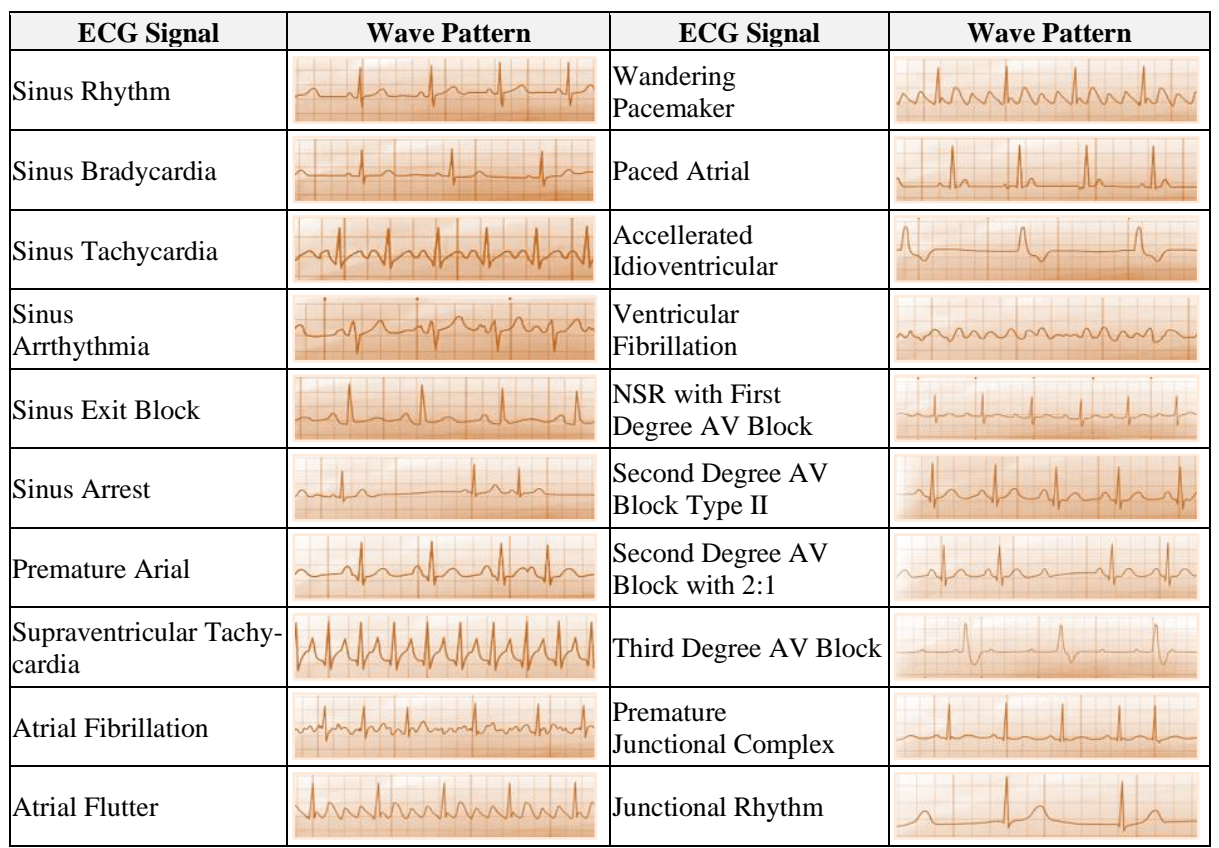


Sinus Rhythm also as normal sinus rhythm (NSR) or regular sinus rhythm (RSR) is the most common adult rhythm with rates ranging from sixty to one hundred per minute. The QRS is most frequently narrow with upright $P$ waves in Lead II. [20] It is a standard heart beat rhythm that compares each time in the first level warning.

Sinus Bradycardia can be well handled by healthy adults at speeds greater than 50 per minute. Due to an optimal volume of cardiac stroke that requires less HR to yield acceptable cardiac output, athletes may routinely be in Sinus bradycard. Sinus bradycardia may also stem from vagal pressure or from Sick Sinus Syndrome. Wait for a short QRS in Lead II with upright $P$ waves.

Sinus Tachycardia is most often the result of such as pain fever, increased sympathetic stimulation, hypovolemia, and increased oxygen demand. The most common symptom of Sinus Tachycardia is elevated sympathetic pressure such as high fever, increased demand for oxygen, and hypovolemia. It usually has a narrow QRS. Often the rate is less than 150 per minute.

Sinus Arrhythmia is most commonly a harmless beat, comman kids and less common in older adults. The repetitive form of this rhythm fluctuates with inspiration or rises in HR and decreases in expiration or HR. In Lead II, a small QRS and upright $P$ waves are expected.

Sinus Exit Block or senatorial blocked sinus impulses are not capable of depolarizing the atria. While on time, the sinus is burning. There is no impulse in the tissue around the SA node. The magnitude of this dysrhythmia is correlated with the block frequency and duration. Each delay is equivalent to several prior $P-P$ intervals.

Sinus Arrest happens when there is no flame on the SA node. Often the resulting delay is not equal to the multiple P-P intervals shown in the Sinus Exit Block. Instead, the cardiac benefit will typically be regulated by an escape pacemaker such as the AV junction like the Sinus exit block, and care is related to the frequency and duration of sinus arrest times.

Premature Atrial Complexes are the result of irritability to the atria resulting in increased atria automaticity initiating an impulse earlier than expected from the SA node. This is a complex that is premature. Expect small QRS and PAC waves that are rounded, notched, pointed or biphasic.

Supraventricular Tachycardia is an alarming pattern usually varying from 170230 per minute. Supraventricular tachycardia's telltale sign is the narrow QRS which determines its supraventricular source and its normal, strong pattern. Because of its very fast rate, the rhythm is most likely not sinus tachycardia. For those who are at rest, the most common supraventricular tachycardia is narrow QRS tachycardia over 150 per minute.

Atrial Fibrillation is a repetitive rhythm of QRS complexes that can be recognized This dysrhythmia is characterized by the Chaotic rhythm pattern and the lack of $P$ waves. Known as fibrillatory waves, the chaotic baseline. It can be seen quickly. After 48 hours, it is particularly significant threat of thrombus formation.

Atrial Flutter results from reentry circuit creation within the atria producing a loop that discharges impulses at 250-350 per minute flutter speed results from reentry circuit 
creation within the atria producing a loop that discharges impulses at 250-350 per minute flutter speed. Most often, the AV junction travels through the ventricles per second or fourth impulse.

Paced Atrial Rhythm benefits from an atrium's electrical timing. Before the $\mathrm{P}$ wave, the vertical bolt. The electronic pacemaker lead produces a low but adequate current repeatedly to begin atria depolarization and the subsequent $P$ wave.

Wandering Pacemaker Rhythm is a supraventricular rhythm with different pulse forming positions resulting in three or more $\mathrm{P}$ waves of variation. With a system of large QRS.

Accellerated Idioventricular Rhythm is a ventricular rhythm that occurs at a rate of 41-100 per minute faster than the typical pacemaker rates expected from the ventricles at 20-40 per minute and less than what is considered to be more than 100 per minute at a tachycardia. It may be due to hypoxia or excessive enhancement for empathy.

Ventricular Fibrillation is a ventricular unstable rhythm. This does not increase in cardiac output. VFib is less than $3 \mathrm{~mm}$ in height, which means less electrical energy in the myocardium, which means less chance of successful defibrillation.

NSR with First Degree AV Block is the product of excessive electrical impulse transmission through the AV junction. A sustained PR interval of more than 20 seconds is the important finding of this pattern. Until alleging a first-degree AV block, the underlying rhythm should be identified and called. This rhythm is a natural sinus rhythm with a first-degree AV block.

Second Degree AV Block Type II is typically due to an irregular block under the AV node due to disrupted supraventricular impulse. Any or more QRS complexes are lowered by fixed PR intervals which do not change.

Second Degree AV Block with 2:1 is a particular second-degree AV block case peculiar with not QRS complex pairing of any alternative $\mathrm{P}$ wave. The interval of PR continues to be constant. This rhythm requires close monitoring due to the risk of poor cardic output associated with a sluggish heart and the ability to progress to the AV block of the third degree.

Third degree AV block is often an alarming condition requiring close monitoring for hemodynamic collapse, ventricular stoppage or asystole development, and other dangerous dysrhythmia. The isolated $\mathrm{P}$ wave ware with an associated QRS complex and erratic PR intervals are significant features of this pattern. A narrow QRS signifies a higher junctional node, while a broad QRS points more in the bundle branches to a sub-nodal stack.

Premature Junctional Complex or PJC occurs within the AV junction from an irritable fixation. A PJC feature involves an incomplete or distorted P wave in lead II and a reduced PR period of less than 0.12 seconds and early or late development of the system.

Junctional Rhythm also is known by the AV interconnection as the junction escape rhythm. The expected rate of pacemakers at the AV crossing is between the fourth and sixty per minute. The rhythm of lead II, which also has the loss of atrial kick, is presented with inverted or missing $\mathrm{P}$ waves. 


\section{Experimental}

\subsection{Electronic sensor kit}

We use the ECG kit circuit for feedback of a real-time signal. [3][6] The medical device industry understands embedded engineering. Embedded engineering has been increasingly complex, making it more difficult for non-technical experts to build fullfeatured medical device models. A new approach to design is therefore required. Graphical System Design is a revolutionary approach to solving design challenges that combine intuitive, graphical programming and flexible, commercial off the shelf hardware while still allowing customization. [1] Graphical System Design blends the skills of an engineering architecture specialist with a technical expert, such as a medical device expert, to drive development.

\subsection{Noise reduction}

Consider the signal $x(m)$ reported in the broadband additive noise $n(m)$ and the context as:

$$
y(m)=x(m)+n(m)
$$

Having regard to the uncorrelated signal and noise, it follows that the autocorrelation matrix of the noisy signal is the sum of the $x(m)$ signal's autocorrelation matrix and $n(m)$ :

$$
R_{y y}=R_{x x}+R_{n n}
$$

Where $R_{y y}, R_{x x}$ and $R_{n n}$ are the noisy signal's autocorrelation matrices, the noise-free signal, and the noise-free signal, respectively, and $R_{x y}$ is the noise-free signal's cross correlation matrix. Substitution of the wiener filters equations (1) and (2) as follows:

$$
\begin{gathered}
w=R_{y y}^{-1} R_{x y} \\
w=\left(R_{x x}+R_{n n}\right)^{-1} R_{x x}
\end{gathered}
$$

This is the perfect linear filter for additive noise reduction. An analysis of the Wiener filter's frequency response provides useful insight into the Wiener filter's operation. The noisy signal $Y(f)$ is given in the frequency domain by

$$
Y(f)=X(f)+N(f)
$$

Where the signal and noise spectra are $X(f)$ and $N(f)$. The frequency-domain Wiener filter is obtained as a signal observed in additive random noise as:

$$
w(f)=\frac{P_{x x}(f)}{P_{x x}(f)+P_{n n}(f)}
$$


Where $P_{x x(f)}$ and $P_{n n(f)}$ are the signal and noise spectra. Divide the numerator and the denominator of equation (6) by the noise spectra $P_{n n(f)}$ and replace the variable $S N R(f)=\frac{P_{x x(f)}}{P_{n n(f)}}$ outputs.

\subsection{Signal and noise}

If the signal frequency and noise do not overlap, a signal is completely recoverable from noise. An example of a noisy signal with a separable signal and noise spectra. The signal and noise in this situation occupy different parts of the frequency spectrum and can be distinguished by a low-pass or high-pass filter. [11] A more common example of a signal and noise process with overlapping spectra can be shown. In this case, it is not possible to separate the signal completely from the noise. However, noise effects can be reduced by using a Wiener filter that attenuates every noisy signal frequency in proportion to an estimate of a signal-to-noise ratio as described in:

$$
w(f)=\frac{\operatorname{SNR}(f)}{\operatorname{SNR}(f)+1}
$$

where SNR is a signal-to-noise ratio measure. Note that the SNR(f) parameter is expressed in terms of the power-spectral ratio, not in the more usual log power ratio terminology. Therefore, $S N R(f)=0$ is equal to $-\infty d B$. The following description of the Wiener filter frequency response $w(f)$ can be deduced from equation (7) in terms of the signal-to-noise ratio. In the case of additive noise, the frequency response of the Wiener filter is a real positive number in the range $0 \leq W(f) \leq 1$. Now consider (a) a noise-free signal $S N R(f)=0$, and (b) an extremely noisy signal $S N R(f)=0$, in the two limiting cases. The filter adds the noise-free frequency portion with little or no attenuation. If $S N R(f)=0, W(f)=0$, at the other extreme. The wiener filter therefore attenuates each frequency component in proportion to an estimate of the signal-to-noise ratio for additive noise. The Wiener filter response variation $W(f)$, with the $S N R(f)$ signal-to-noise ratio. An alternative example of the differences in the $S N R(f)$ response of the wiener filter frequency. This shows the similarities in an integrated white noise interference between the wiener filter frequency response and the signal spectrum Note that the Wiener filter frequency response is also high at a spectral peak of the signal spectrum, where the $S N R(f)$ is relatively high, and little attenuation is applied to the filter. The signal-tonoise ratio is small at a signal trough and so is the output of the Wiener filter. Therefore, the Wiener filter response broadly follows the signal spectrum for additive white noise.

\subsection{Signal reduction}

The correlation of the sample corresponds to the predicted value of past samples. It's obsolete and can be dropped. The uncorrelated part that represents the prediction error or residual signal is then left to us. The residual signal frequency spectrum is less than the initial signal. For expression, it requires fewer bits. By applying Huffman coding to the residual signal, we can further through the results. They define briefly two ECG reduction algorithms using residual differentiation as follows: 


$$
\mathrm{x}^{\prime}(\mathrm{nT})=\sum_{\mathrm{k}=1}^{\mathrm{p}} \mathrm{a}_{\mathrm{k}} \mathrm{x}(\mathrm{nT}-\mathrm{kT})
$$

where the original data is $x(n T) . x^{\prime}(n T)$ is the number of samples predicted, $P$ is the number of samples predicted. The $a_{k}$ parameters are selected to minimize the expected mean squared error $E\left[\left(x-x^{\prime}\right)^{2}\right]$ when $p=1$, we select $a_{l}=1$ and we take the first signal difference. The reference value estimator consists a linear mixture of past and future measurements in interpolation. The predictor results indicated a sufficient second order estimator. The interpolator takes only a selection from the past and from the future.

$$
x^{\prime}(n)=\operatorname{ax}(n T-T)+b x(n T+T)
$$

where the a and b coefficients are calculated by decreasing the mean squared error predicted. The prediction and interpolation residuals are encoded using a modified Huffman coding scheme, where the frequent set consists of a few quantized zero-centered levels.

\subsection{Integration component diagnosis (ICD) using ECG signal recognition}

We are designing this algorithm for rapid extraction of features and fast recognition. Principle Component Analysis is typically a statistical method using an orthogonal transformation to translate a set of observations of theoretically associated variables into a set of values of linearly uncorrelated variables called Integration Component Diagnosis (ICD). [16][24] In the ECG signal, it is an innovative approach that relies on numerical speed. ICD will generally consider parallel dimension while simultaneous signal extraction feature. Standard algorithm methods assign data vectors to the (2D) ${ }^{2}$ PCA's own space combination transforming the projected new data with nonGaussianity ICD, which is high-dimensional pattern recognition. The Non-Gaussianity is performed directly on the own matrix of the signal recognition feature. The original data is projected directly into the final space of their own. Repeated high-dimensional data operations are avoided and the amount of calculation is reduced. High performance approach is the combination of two algorithms. (2D) ${ }^{2}$ PCA and alternative 2DPCA operate only in the pattern line and column direction. (2D) ${ }^{2} \mathrm{PCA}$ is provided with an optimal matrix $X$ of the set of training patterns that reflects information between pattern rows, then projects $\mathrm{m}$ by $\mathrm{n}$ dimensions $A$ to $X$ to produce $\mathrm{m}$ with $\mathrm{d}$ matrix $Y=A X$. Likewise, the alternative 2DPCA learns optimum matrix $Z$ reflecting the data between dimensional columns, and then projects $A$ to $Z$ to produce a $q$ by $n$ matrix $B=Z^{T} A$. The projection matrices $X$ and $Z$ are given here below, provided we get projection matrices $X$ and $Z$, which are projected $m$ at $n$ dimension $A$ simultaneously to $X$ and $Z$ and give a $q$ at $d$ matrix $C$.

$$
\mathrm{C}=\mathrm{Z}^{\mathrm{T}} \mathrm{AX}
$$

The matrix $C$ is also referred to as the coefficient matrix in the data representation, which can be used to reconstruct the original signal $A$.

$$
\widehat{\mathrm{A}}=\mathrm{ZCX}^{\mathrm{T}}
$$


when used for signal recognition, matrix $C$ is also referred to as a function matrix. Depending on the estimation of each learning information element. $A_{k}$ by $k=1,2, \ldots, M$ on $X$ and $Z$, we obtain the $C_{k}$ matrix training feature by $C=1,2, \ldots, M$. Given the recognition of the test signal. Here the distance between $C$ and $C_{k}$ is defined by the following:

$$
\mathrm{d}\left(\mathrm{C}, \mathrm{C}_{\mathrm{k}}\right)=\sqrt{\sum_{\mathrm{i}=1}^{\mathrm{q}} \sum_{\mathrm{j}=1}^{\mathrm{d}}\left(\mathrm{C}^{(\mathrm{i}, \mathrm{j})}-\mathrm{C}_{\mathrm{k}}^{(\mathrm{i}, \mathrm{j})}\right)^{2}}
$$

The independent component is estimated by focusing on non-Gaussianity. Since it is presumed that each underlying origin is not normally distributed, one way to remove the components is by pressuring each of them to separate themselves as far as possible from the normal distribution. Negentropy may be used to predict non-Gaussianity. In short, negentropy is a distance measurement from normality that is described by:

$$
\mathrm{N}(\mathrm{X})=\mathrm{H}\left(\mathrm{X}_{\text {Gaussian }}\right)-\mathrm{H}(\mathrm{X})
$$

If $X$ is a random variable considered to be non-Gaussian, $H(X)$ is the entropy.

$$
\mathrm{H}(\mathrm{X})=-\sum_{\mathrm{X}} \mathrm{P}(\mathrm{x}) \log (\mathrm{x})
$$

where $X_{\text {Gaussian }}$ is the entropy of a Gaussian random vector variable with a matrix of covariance equal to $X$. The distribution that has the maximum entropy for a given covariance matrix is the Gaussian distribution. Negentropy is therefore a purely positive measure of non-Gaussianity. However, it is difficult to calculate negentropy using equation (13) which is why approximations are being used.

$$
N(V)=E(j(V))-E(j(U))^{2}
$$

Where $V$ is a standardized non-Gussian random variable for zero mean, $U$ is a standardized Gussian random variable and $\phi($.$) is a non-quadratic function. Set initialize W_{i}$ algorithm from probability after some manipulation to the first step.

$$
\begin{gathered}
\mathrm{W}_{\mathrm{i}}^{+}=\mathrm{E}\left(\mathrm{j}^{\prime}\left(\mathrm{w}_{\mathrm{i}}^{\mathrm{T}} \mathrm{X}\right)\right) \mathrm{w}_{\mathrm{i}}-\mathrm{E}\left(\mathrm{xj}\left(\mathrm{w}_{\mathrm{i}}^{\mathrm{T}} \mathrm{X}\right)\right) \\
\mathrm{W}_{\mathrm{i}}=\frac{\mathrm{w}_{\mathrm{i}}^{+}}{\left\|\mathrm{w}_{\mathrm{i}}^{+}\right\|}, \mathrm{i}^{1} \neq 1
\end{gathered}
$$

if $i \neq 1$ then calculate in the next step

$$
\mathrm{W}_{\mathrm{i}}^{+}=\mathrm{W}_{\mathrm{i}}-\sum_{\mathrm{j}=1}^{\mathrm{i}-1} \mathrm{~W}_{\mathrm{i}}^{\mathrm{T}} \mathrm{W}_{\mathrm{j}}, \mathrm{i}^{1} \neq 1
$$

Return to equation (16) if not converged. If consideration is not given then go back to Initialize $w_{i}$ again by $i=i+1$ until all components have been extracted where $w_{i}$ is a $W$ mixing matrix column-vector, $w_{i}$ is a temporary parameter used to measure $w_{i}$. Before normalization, it is the new $w_{i}, \phi($.$) is the derivative of \phi($.$) and E($.$) is the mean$ value expected. Once a given $w_{i}$ has converged, the next one $\left(w_{i}+1\right)$ and all those previously derived with equations (17) and (18) must be made orthogonal to it in order to distinguish the new component from the previous one. 


\subsection{Optimization algorithm (OA) using ECG signal recognition}

The particle $i$ in a D-dimensional space is defined as:

$$
\mathrm{X}_{\mathrm{i}}=\left(\mathrm{x}_{\mathrm{i} 1}, \mathrm{x}_{\mathrm{i} 2}, \mathrm{x}_{\mathrm{i} 3}, \ldots \mathrm{x}_{\mathrm{iD}}\right)
$$

and each particle retains its previous best location memory. $i_{\text {th }}$ particle's best previous location can be interpreted as:

$$
P_{i}=\left(p_{i 1}, p_{i 2}, p_{i 3}, \ldots p_{i D}\right)
$$

and $i_{t h}$ particle velocity is represented as:

$$
\mathrm{V}_{\mathrm{i}}=\left(\mathrm{v}_{\mathrm{i} 1}, \mathrm{v}_{\mathrm{i} 2}, \mathrm{v}_{\mathrm{i} 3}, \ldots \mathrm{v}_{\mathrm{iD}}\right)
$$

The global best is considered the particle location with the greatest fitness value for the whole race. The best global particle in the population among all the particles is defined by:

$$
P_{g}=\left(p_{g 1}, p_{g 2}, p_{g 3}, \ldots p_{g D}\right)
$$

The location of the velocity change made by the previous best location of the object is considered the component of perception, and the position of the velocity adjustment is called the social component using the global best.

$$
\begin{gathered}
\mathrm{v}_{\mathrm{id}}^{1}(\mathrm{t}+1)=\omega \mathrm{v}_{\text {id }}(\mathrm{t})+\mathrm{n}_{1} * \operatorname{rand}() *\left(\mathrm{p}_{\mathrm{id}}(\mathrm{t})-\mathrm{x}_{\mathrm{id}}(\mathrm{t})\right) \\
\mathrm{v}_{\mathrm{id}}^{2}=\mathrm{n}_{2} * \operatorname{rand}(\mathrm{C})\left(\mathrm{p}_{\mathrm{gd}}(\mathrm{t})-\mathrm{x}_{\mathrm{id}}(\mathrm{t})\right) \\
\mathrm{v}_{\mathrm{id}}=\mathrm{v}_{\mathrm{id}}^{1}+\mathrm{v}_{\mathrm{id}}^{2}, \mathrm{x}_{\mathrm{id}}(\mathrm{t}+1)=\mathrm{x}_{\text {id }}(\mathrm{t})+\mathrm{v}_{\text {id }}(\mathrm{t})
\end{gathered}
$$

where the weight of inertia is equal to that of inertia, $\eta 1$ and $\eta 2$ are positive constants of acceleration. The velocity vector drives the process of optimization and reflects data that has been exchanged socially. It operates as follows: initialize the $P(t)$ swarm of particles so that the $X i(t)$ position of each $\operatorname{PiEP}(t)$ particle is random in the hyperspace, with $t=0$., evaluate the $F(X i(t))$ performance of each particle using its current $X i(t)$ location. Contrast the individual's performance to its best performance so far: if $F(X i(t))<p i d$ then (a) $p_{i d}=F(X i(t))$ and (b) $P i=X i(t)$, contrast the quality of each particle to the best global particle if $F(X i(t))<p_{g d}$ then (a) $p_{g d}=F(X i(t))$ (b) $P_{g}=X i(t)$, change the vector of each particle as equation (25). The last term is the component of society. Next step moves the particle to a new location. $x_{i d}(t+1)=x_{i d}(t)+v_{i d}(t)$ and $t=t+1$, repeat output calculation. The further away a particle is from the best location in the global and its own best solution to return visit, the greater the change in velocity to move the particle back to the best solutions. 


\section{$4 \quad$ Result of Experimental}

We test 200 signals that are multiple ECG patterns and repeat the test 5 times. In computer programming, we are developing with the $\mathrm{C}++$ language. Experiment results in Table 3-4.

Table 3. Signal Idenify Recognition

\begin{tabular}{|l|c|c|c|}
\hline \multicolumn{1}{|c|}{ ECG Signal } & $\begin{array}{c}\text { Automata } \\
\text { Average Time } \\
\text { (seconds) }\end{array}$ & $\begin{array}{c}\text { OA Average } \\
\text { Time (seconds) } \\
\text { (seconds) }\end{array}$ & $\begin{array}{c}\text { ICD Average } \\
\text { Time (seconds) }\end{array}$ \\
\hline Sinus Rhythm & 5.547 & 3.918 & 3.517 \\
\hline Sinus Bradycardia & 6.391 & 5.419 & 5.118 \\
\hline Sinus Tachycardia & 6.774 & 5.554 & 5.069 \\
\hline Sinus Arrthythmia & 7.417 & 6.765 & 5.954 \\
\hline Sinus Exit Block & 5.219 & 4.316 & 3.849 \\
\hline Sinus Arrest & 5.918 & 4.542 & 3.875 \\
\hline Premature Arial & 4.985 & 4.071 & 3.519 \\
\hline Supraventricular Tachycardia & 6.013 & 5.572 & 4.985 \\
\hline Atrial Fibrillation & 5.182 & 4.549 & 3.914 \\
\hline Atrial Flutter & 6.562 & 6.228 & 5.092 \\
\hline Wandering Pacemaker & 4.968 & 3.935 & 3.157 \\
\hline Paced Atrial & 6.106 & 5.648 & 4.845 \\
\hline Accellerated Idioventricular & 5.154 & 3.792 & 2.993 \\
\hline Ventricular Fibrillation & 3.289 & 2.811 & 2.013 \\
\hline NSR with First Degree AV Block & 5.118 & 4.175 & 3.379 \\
\hline Second Degree AV Block Type II & 6.216 & 5.851 & 4.914 \\
\hline Second Degree AV Block with 2:1 & 5.342 & 4.759 & 3.815 \\
\hline Third Degree AV Block & 6.935 & 6.297 & 5.018 \\
\hline Premature Junctional Complex & 5.195 & 4.285 & 3.092 \\
\hline Junctional Rhythm & 6.491 & 6.075 & 4.984 \\
\hline
\end{tabular}

The result is the experimentation of Integration Component Diagnosis (ICD) algorithm more than the Automata matching and Optimization Algorithm (OA) algorithm is reliability and fastness in signal recognition. The hypothesis of this study, Integration Component Diagnosis (ICD) uses 2D 2 PCA quickly in functional extraction in combination with simple, independent, noise-reduction component analysis and separate processing recognition. Such algorithms are a simple and highly effective solution that takes less time than the Automata match the signal is sufficient to transmit to the patient's device We're using two layers of alert. The first level, this system will alert you when your heartbeat is different from the Sinus Rhythm wave pattern [13], which is the standard pattern of a normal human. This machine can sense an abnormal signal at the second level. [14] Though, two levels will spend less than 12 seconds of time. Because, the Heart Failure is the phase of emergency critical for the patient. 
Table 4. Average Percentage of Analyzed

\begin{tabular}{|l|c|c|}
\hline \multicolumn{1}{|c|}{ ECG Signal } & $\begin{array}{c}\text { ICD analyzed faster than } \\
\text { Automata }(\%)\end{array}$ & $\begin{array}{c}\text { ICD analyzed faster than } \\
\text { OA (\%) }\end{array}$ \\
\hline Sinus Rhythm & 36.60 & 29.37 \\
\hline Sinus Bradycardia & 19.92 & 15.21 \\
\hline Sinus Tachycardia & 25.17 & 18.01 \\
\hline Sinus Arrthythmia & 19.72 & 8.79 \\
\hline Sinus Exit Block & 26.25 & 17.30 \\
\hline Sinus Arrest & 34.52 & 23.25 \\
\hline Premature Arial & 29.41 & 18.34 \\
\hline Supraventricular Tachycardia & 17.10 & 7.33 \\
\hline Atrial Fibrillation & 24.47 & 12.22 \\
\hline Atrial Flutter & 22.40 & 5.09 \\
\hline Wandering Pacemaker & 36.45 & 20.79 \\
\hline Paced Atrial & 20.65 & 7.50 \\
\hline Accellerated Idioventricular & 41.93 & 26.43 \\
\hline Ventricular Fibrillation & 38.80 & 14.53 \\
\hline NSR with First Degree AV Block & 33.98 & 18.43 \\
\hline Second Degree AV Block Type II & 20.95 & 5.87 \\
\hline Second Degree AV Block with 2:1 & 28.58 & 10.91 \\
\hline Third Degree AV Block & 27.64 & 9.20 \\
\hline Premature Junctional Complex & 40.48 & 17.52 \\
\hline Junctional Rhythm & 23.22 & 6.41 \\
\hline
\end{tabular}

The study would measure the 200 signals from different ECG signals. Integration Component Diagnosis (ICD) is capable of an unusual accuracy of recognition than the Automata Matching Process 17.10-41.93 average percentage and spends less time than the other 29.37 average percentage method and can alert within 12 seconds period. It cannot be detected in a pattern that is different from without an ECG signal being recognized. The signal is used in the experiment to recognize 20 patterns of the ECG signal. (Figure 3)

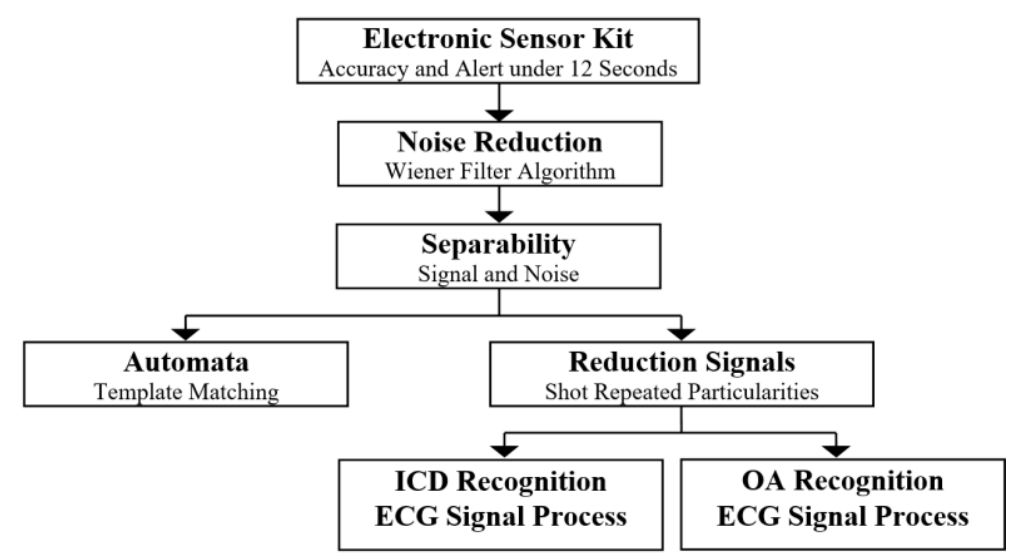

Fig. 3. Compare of Experimental Framework the Automata Matching, OA and ICD 


\section{Conclusion}

The Electronic Sensor Kit includes support for a wide range of signal conversion panels, an extensive signal processing algorithm library including Integration Component Diagnosis (ICD), Optimization Algorithm (OA), convolution, low/high-pass [11], and bandpass filters, and a novelty platform. Although our review has a limited scope, ECG analysis remains an area of intense research. It could be argued. In recent decades, methodologies for pattern recognition have been widely used in this field. While significant attempts have been and are currently being made to identify the best characteristics suited to the various applications, the emerging device of human identity has not yet achieved a defined status and it is expected that further research by the scientific community will be oriented towards this area.

\section{Acknowledgement}

This research was supported by Chandrakasem Rajabhat University. Thanks, our colleagues from Chandrakasem Rajabhat University who provided insight and expertise that greatly assisted the research.

\section{$7 \quad$ References}

[1] Abubakar Adam, Adamu Abubakar, Murni Mahmud. (2019). Sensor Enhanced Health Information Systems: Issues and Challenges. International Journal of Interactive Mobile Technologies, Vol 13, No 01, Pages 99-114. https://doi.org/10.3991/ijim.v13i01.7037

[2] Ahilan Appathurai, J. Jerusalin Carol, C. Raja, S. N. Kumar, Sujatha Krishnamoorthy. (2019). A study on ECG signal characterization and practical implementation of some ECG characterization techniques. Measurement, Volume 147, December 2019, Article 106384. https://doi.org/10.1016/j.measurement.2019.02.040

[3] Anchana Muankid, Mahasak Ketcham. (2019). The Real-time Electrocardiogram Signal Monitoring System in Wireless Sensor Network. International Journal of Online and Biomedical Engineering, Vol 15, No 02, Pages 4-20. https://doi.org/10.3991/ijoe.v15i02.9422

[4] Anthony G. Pompa, Peter LaRossa, Lee B. Beerman, Yoshimi Sogawa, Gaurav Arora. (2019). Utility of ECGs in the pediatric emergency department for patients presenting with a seizure. The American Journal of Emergency Medicine. Available online 20 November 2019. https://doi.org/10.1016/j.ajem.2019.11.016

[5] Aykut Diker, Engin Avci, Erkan Tanyildizi, Mehmet Gedikpinar. (2019). A Novel ECG Signal Classification Method using DEA-ELM. Medical Hypotheses, In press, journal preproof, Available online 13 December 2019, Article 109515. https://doi.org/10.1016/j. mehy.2019.109515

[6] Carmen Camara, Pedro Peris-Lopez, Lorena Gonzalez-Manzano, Juan Tapiador. Real-time electrocardiogram streams for continuous authentication. Applied Soft Computing, Volume 68, July 2018, Pages 784-794. https://doi.org/10.1016/j.asoc.2017.07.032

[7] Chunqiang Qian, Honghong Su, Helong Yu. (2019). Local means denoising of ECG signal. Biomedical Signal Processing and Control, Volume 53, August 2019, Article 10157. https://doi.org/10.1016/j.bspc.2019.101571 
[8] Emrah Bozbeyoğlu, Emre Aslanger, Özlem Yıldırımtürk, Barış Şimşek, Muzaffer Değertekin. (2018). An algorithm for the differentiation of the infarct territory in difficult to discern electrocardiograms. Journal of Electrocardiology, Volume 51, Issue 6, November-December 2018, Pages 1055-1060. https://doi.org/10.1016/j.jelectrocard.2018.09.006

[9] Gherardo Finocchiaro, Nabeel Sheikh, Elena Biagini, Michael Papadakis, Iacopo Olivotto. (2019). The electrocardiogram in the diagnosis and management of patients with hypertrophic cardiomyopathy. Heart Rhythm, In press, corrected proof, Available online $10 \mathrm{Au}-$ gust 2019. https://doi.org/10.1016/j.hrthm.2019.07.019

[10] Henry J. Lin, Yueh-Tze Lan, Michael J. Silka, Nancy J. Halnon, Ruey-Kang Chang. Home use of a compact, 12-lead ECG recording system for newborns. Journal of Electrocardiology, Volume 53, March-April 2019, Pages 89-94. https://doi.org/10.1016/j.jelectrocard.2019.01.086

[11] Jonas Isaksen, Remo Leber, Ramun Schmid, Hans-Jakob Schmid, Roger Abächerli. (2017). Quantification of the first-order high-pass filter's influence on the automatic measurements of the electrocardiogram. Computer Methods and Programs in Biomedicine, Volume 139, February 2017, Pages 163-169. https://doi.org/10.1016/j.cmpb.2016.11.003

[12] Kandala N. V. P. S. Rajesh, Ravindra Dhuli. (2017). Classification of ECG heartbeats using nonlinear decomposition methods and support vector machine. Computers in Biology and Medicine, Volume 87, 1 August 2017, Pages 271-284. https://doi.org/10.1016/j.compbiomed.2017.06.006

[13] Luca Mesin. (2017). Heartbeat monitoring from adaptively down-sampled electrocardiogram. Computers in Biology and Medicine, Volume 84, 1 May 2017, Pages 217-225. https://doi.org/10.1016/j.compbiomed.2017.03.023

[14] M. K. Moridani, M. Abdi Zadeh, Z. Shahiazar Mazraeh. (2019). An Efficient Automated Algorithm for Distinguishing Normal and Abnormal ECG Signal. IRBM, Volume 40, Issue 6, December 2019, Pages 332-340. https://doi.org/10.1016/j.irbm.2019.09.002

[15] Margherita Padeletti, Giuseppe Bagliani, Roberto De Ponti, Fabio M. Leonelli, Emanuela T. Locati. (2019). Surface Electrocardiogram Recording: Baseline 12-lead and Ambulatory Electrocardiogram Monitoring. Cardiac Electrophysiology Clinics, Volume 11, Issue 2, June 2019, Pages 189-201. https://doi.org/10.1016/j.ccep.2019.01.004

[16] Mounaim Aqil, Atman Jbari, Abdennasser Bourouhou. (2017). ECG Signal Denoising by Discrete Wavelet Transform. International Journal of Online and Biomedical Engineering, Vol 13, No 09, Pages 51-68. https://doi.org/10.3991/ijoe.v13i09.7159

[17] Rajani Akula, Hamdi Mohamed. (2019). Automation algorithm to detect and quantify Electrocardiogram waves and intervals. Procedia Computer Science, Volume 151, 2019, Pages 941-946. https://doi.org/10.1016/j.procs.2019.04.131

[18] Rajender Singh, Jeremy J. Murphy. (2018). Electrocardiogram and arrhythmias. Anaesthesia \& Intensive Care Medicine, Volume 19, Issue 6, June 2018, Pages 322-325. https://doi.org/10.1016/j.mpaic.2018.03.014

[19] S. Kota, C. B. Swisher, T. Al-Shargabi, N. Andescavage, R. B. Govindan. (2017). Identification of QRS complex in non-stationary electrocardiogram of sick infants. Computers in Biology and Medicine, Volume 87, 1 August 2017, Pages 211-216. https://doi.org/10.1016 /..compbiomed.2017.05.033

[20] Safri, Wan Nishfa Dewi, Erwin. (2018). Analysis of electrocardiogram recording lead II in patients with cardiovascular disease. Enfermería Clínica, Volume 29, Supplement 1, March 2019, Pages 23-25. https://doi.org/10.1016/j.enfcli.2018.11.011

[21] Sara Mihandoost, Mehdi Chehel Amirani. (2017). Cyclic spectral analysis of electrocardiogram signals based on GARCH model. Biomedical Signal Processing and Control, Volume 31, January 2017, Pages 79-88. https://doi.org/10.1016/j.bspc.2016.07.012 
[22] Teresa Rocha, Simão Paredes, Ramona Cabiddu, Ricardo Couceiro, Paulo Carvalho, Jorge Henriques. (2019). A Tool for ECG Analysis as a Module of a Tele-Monitoring System. International Journal of Online and Biomedical Engineering, Vol 13, No 09, Pages 64-67. http://dx.doi.org/10.3991/ijoe.v12i04.5260. https://doi.org/10.1109/expat.2015.7463235

[23] Tomas Novotny, Raymond Bond, Irena Andrsova, Lumir Koc, Marek Malik. (2017). The role of computerized diagnostic proposals in the interpretation of the 12-lead electrocardiogram by cardiology and non-cardiology fellows. International Journal of Medical Informatics, Volume 101, May 2017, Pages 85-92. https://doi.org/10.1016/j.ijmedinf.2017.02.007

[24] Weiyi Yang, Yujuan Si, Di Wang, Gong Zhang. (2019). A Novel Method for Identifying Electrocardiograms Using an Independent Component Analysis and Principal Component Analysis Network. Measurement, In press, journal pre-proof, Available online 5 December 2019, Article 107363. https://doi.org/10.1016/j.measurement.2019.107363

\section{Author}

Kittimasak Naijit works as a lecturer in Multimedia Technology, Faculty of Science at Chandrakasem Rajabhat University, Bangkok, Thailand, 10900. He has got the bachelor's 2 degrees in Computer Education and Computer Engineering, and graduated with a master's degree in Computer Technology, King Mongkut's University of Technology North Bangkok (KMUTNB). His research interests are in the area of artificial intelligence, robotics, information technology systems and multimedia technology.

Article submitted 2019-12-13. Resubmitted 2020-01-11. Final acceptance 2020-01-13. Final version published as submitted by the authors. 